\title{
Cell Condensation Triggers the Differentiation of Osteoblast Precursor Cells to Osteocyte-Like Cells
}

\author{
Jeonghyun Kim and Taiji Adachi* \\ Biomechanics Laboratory, Institute for Frontier Life and Medical Sciences, Kyoto University, Kyoto, Japan
}

Though the three-dimensional (3D) in vitro culture system has received attention as a powerful tool for conducting biological research, in vitro bone formation and osteocyte differentiation studies have mostly been based on results obtained using two-dimensional (2D) culture systems. Here, we introduced a rotatory culture system to fabricate

OPEN ACCESS

Edited by:

Martin James Stoddart, AO Research Institute, Switzerland

Reviewed by:

Matt Prideaux,

Indiana University-Purdue University Indianapolis, United States Arnaud Scherberich,

University Hospital of

Basel, Switzerland Astrid Bakker,

Vrije Universiteit University Amsterdam, Netherlands

*Correspondence:

Taiji Adachi

adachi@infront.kyoto-u.ac.jp

Specialty section:

This article was submitted to Tissue Engineering and Regenerative

Medicine,

a section of the journal Frontiers in Bioengineering and Biotechnology

Received: 31 July 2019 Accepted: 09 October 2019 Published: 23 October 2019

Citation:

Kim J and Adachi T (2019) Cell

Condensation Triggers the

Differentiation of Osteoblast Precursor

Cells to Osteocyte-Like Cells.

Front. Bioeng. Biotechnol. 7:288.

doi: 10.3389/fbioe.2019.00288
3D spheroids, using mouse osteoblast precursor cells. These spheroids, incubated for 2 days without chemical induction by osteogenic supplements, exhibited notably up-regulated osteocyte marker levels; osteoblast marker levels were down-regulated, as compared to those of the conventional 2D monolayer model. The cell condensation achieved with the 3D spheroid structure triggered a greater level of differentiation of osteoblast precursor cells into osteocyte-like cells than that observed during chemical induction. Our study might imply that osteoblasts proliferate and become condensed at the targeted bone remodeling site, because of which osteoblasts achieved the capability to differentiate into osteocytes in vivo.

Keywords: spheroids, differentiation, osteocyte, osteoblast, rotatory culture, cell condensation

\section{INTRODUCTION}

Three-dimensional (3D) culture systems known as organoids have recently been in focus, as they have been used to recapitulate the morphogenesis and functioning of various types of organs (Sasai, 2013; Rossi et al., 2018), such as the small intestine (Spence et al., 2011), liver (Takebe et al., 2013), stomach (McCracken et al., 2014), and lungs (Dye et al., 2015). The 3D structure of cells inducing the appropriate cell-cell interactions during the organ formation process has become known; hence, the demand for the generation of a $3 \mathrm{D}$ in vitro culture system by researchers studying human development, disease, and drug screening has increased (Rossi et al., 2018). However, the structural effects and configurations of cells in the 3D culture system, and especially cellular behavior, including differentiation capability, are not fully understood yet. Although a conventional two-dimensional (2D) culture system has greatly enabled us to understand cellular behavior, including gene expression and homeostasis, it might alter several intracellular signaling pathways, as compared to those present in vivo, thereby causing distinct biological outcomes. Hence, a more detailed study using the 3D culture system would be required to understand cellular behavior in complex native structures.

While the $3 \mathrm{D}$ culture system is regarded as a promising tool for performing in vitro biological studies, the introduction of the $3 \mathrm{D}$ model is also thought to have influenced the study of in vitro bone formation. The bone is composed of mineralized collagen fibrils induced via the formation 
of apatite crystals (Nair et al., 2013), and it is also known as a dynamic tissue that undergoes remodeling with osteoclasts and osteoblasts throughout the lifespan of a mammal (Weatherholt et al., 2012). Osteocytes comprise $\sim 95 \%$ of bone cells that are embedded inside the mineralized bone matrix (Adachi et al., 2009; Bonewald, 2011). Due to the difficulty in retaining the osteocyte-likeness in vitro after osteocyte isolation, in vitro models utilizing osteocytes are fewer in number, whereas osteoblasts have been utilized as a surrogate. However, current in vitro bone formation and osteocyte differentiation studies have mostly been carried out with the $2 \mathrm{D}$ model, using the chemical induction process. The role of chemical supplements, such as ascorbic acid and $\beta$-glycerophosphate in the osteogenic differentiation process was successfully revealed with the use of this model (Malaval et al., 1994; Coelho and Fernandes, 2000; Buttery et al., 2004). Moreover, the conventional methods allowed osteoprogenitor cells to induce osteogenic differentiation over 3-4 weeks (Quarles et al., 1992; Wang et al., 1999). As a result of this long-term cultivation of osteoprogenitor cells, the proliferated cells formed a highly localized pile of confluent cells, which resulted in the bone nodule having a 3D domeshaped structure (Bhargava et al., 1988; Kawai et al., 2019). Inside the bone nodule, the cells are induced to differentiate into osteoblasts, and these cells secreted a highly organized collagen matrix and further mineralized the deposited extracellular matrix (ECM), including alkaline phosphatase (ALP). Furthermore, osteocyte-like cells were observed inside this in vitro bone nodule (Kawai et al., 2019). These in vitro results, however, are yet to sufficiently mimic the in vivo bone formation with regard to the level of differentiation and induction time (Blair et al., 2017). Hence, a paradigm shift is required in a new in vitro osteocyte model, such as the $3 \mathrm{D}$ culture system. The development of the new in vitro $3 \mathrm{D}$ osteocyte culture model is expected to provide new insights into the biology of osteocytes and the utilization of this information to achieve well-organized bone formation. Apart from its application in vitro, the $3 \mathrm{D}$ osteocyte model is also predicted to become a potential tool for tissue-engineered regenerative medicine, owing to the ability to transplant them. We hereby postulate that the cell condensation achieved with the $3 \mathrm{D}$ structure provides an appropriate cellECM interaction for cells and further plays a significant role in osteogenic differentiation.

In this study, it is proposed that the cell condensation in the 3D culture system efficiently induced osteogenic differentiation. Particularly, we fabricated 3D bone spheroids using preosteoblast cells under rotatory culture conditions, without introducing any chemical supplements. By using this model, we evaluated the differentiation capacity of the spheroids and further attempted to highlight the driving force for triggering in vitro osteogenic differentiation.

\section{MATERIALS AND METHODS}

\section{Cell Culture}

In this study, we utilized the murine pre-osteoblast cell line MC3T3-E1. Cells were cultured in MEM- $\alpha$ (Gibco), consisting of $10 \%$ fetal bovine serum (Gibco), and 1\% antibiotic-antimycotic
(Gibco) solution in a humidified incubator at $37^{\circ} \mathrm{C}$, in the presence of $5 \% \mathrm{CO}_{2}$. We carried out passaging when the confluency of the cells became up to $80-90 \%$. To prepare an osteogenic induction medium, we subcultured cells with osteogenic supplements containing $50 \mu \mathrm{g} / \mathrm{ml}$ ascorbic acid and $10 \mathrm{mM} \beta$-glycerophosphate. To prepare the $2 \mathrm{D}$ monolayer sample, 200,000 cells were subcultured on a $35 \mathrm{~mm}$ culture dish (cell density: 208 cells $/ \mathrm{mm}^{2}$ ), to make it fully confluent after a 2-days incubation period.

\section{Fabrication of Spheroids}

We fabricated cell spheroids using MC3T3-E1 cells, by optimizing the protocol described by Furukawa et al. (2001). First, 1,000,000 cells were subcultured with the culture medium in a 6-well ultra-low attachment plate (Corning Coaster) (cell density: 1,040 cells $/ \mathrm{mm}^{2}$ ). The culture plate containing the subcultured cells was then subjected to a rotational culture process at $70 \mathrm{rpm}$ for $8 \mathrm{~h}$, in a humidified incubator at $37^{\circ} \mathrm{C}$, in the presence of $5 \% \mathrm{CO}_{2}$. The spheroids formed were further incubated in a stationary culture process for 2-4 days.

\section{Real Time-PCR}

The samples were collected and lysed in $1 \mathrm{ml}$ of Isogen II (Nippon Gene) for RNA extraction. After adding $50 \mu \mathrm{l}$ of $\mathrm{p}$ Bromoanisole (Nacalai Tesque), the samples were centrifuged at $12,000 \mathrm{~g}$ for $10 \mathrm{~min}$ at $4^{\circ} \mathrm{C}$. The supernatants were separated from the mixture and transferred to new microtubes, into which the same amounts of $70 \%$ ethanol were added. After mixing the tube well, the mixtures were transferred into the spin cartridge (PureLink RNA Mini kit; Invitrogen). The samples were subjected to the centrifugation process, followed by washing and drying, according to the manufacturer's protocol. An appropriate amount of RNase free water was then added, depending on the sample amount. To perform cDNA synthesis and real-time PCR (RT-PCR), the Transcriptor Universal cDNA Master (Roche) and PowerUp SYBR Green Master Mix (ThermoFisher) were used, respectively. As shown in Table 1, all the PCR primer sequences were designed by Invitrogen.

All the markers shown below have been classified according to the method described in previous studies (Bonewald, 2011; Capulli et al., 2014). We examined runt related transcription factor 2 (Runx2) and osterix (Osx) for osteoprogenitor markers, while alkaline phosphatase $(A l p)$ and collagen type I alpha 1 chain (Col1a1) acted as pre-osteoblast markers. To identify osteoblast markers, we examined the gene expression of distalless homebox $5(D l x 5)$, bone sialoprotein $(B s p)$, and osteocalcin $(\mathrm{Ocn})$. With regard to osteocyte markers, we divided the markers into three groups, depending on osteocyte maturity, as markers of osteoid osteocytes, mineralizing osteocytes, and mature osteocytes. Osteopontin $(O p n)$ and the phosphate regulating endopeptidase homolog X-linked (Phex) were investigated, to check the gene expression levels of osteoid osteocyte markers. Among mineralizing osteocyte markers, we examined the dentin matrix protein 1 (Dmp1), capping actin protein, and gelsolinlike $(C a p G)$ protein, whereas sclerostin (Sost) was considered as a marker of mature osteocytes. All the gene expression 
TABLE 1 | Primer list.

\begin{tabular}{|c|c|c|c|}
\hline Gene & Forward primer & Reverse primer & Amplicon size (bp) \\
\hline Gapdh & TGTTCCTACCCCCAATGTGT & GGTCCTCAGTGTAGCCCAAG & 137 \\
\hline Runx2 & CAGTCCCAACTTCCTGTGCT & TACCTCTCCGAGGGCTACAA & 94 \\
\hline Osx & CTCCATCTGCCTGACTCCTT & GGGACTGGAGCCATAGTGAG & 91 \\
\hline Alp & GCTGATCATTCCCACGTIIT & ACCATATAGGATGGCCGTGA & 120 \\
\hline Col1a1 & CGTGCAATGCAATGAAGAAC & TCCCTCGACTCCTACATCTTCT & 118 \\
\hline$D / x 5$ & CCAGCCAGAGAAAGAAGTGG & TTGGTTTACCATTCACCATCC & 56 \\
\hline Bsp & GGAGGCAGAGAACTCCACAC & TTCTGCATCTCCAGCCTTCT & 130 \\
\hline Ocn & GCGCTCTGTCTCTCTGACCT & CGCCGGAGTCTGTTCACTAC & 107 \\
\hline Opn & CCCGGTGAAAGTGACTGATT & GGCTITCATTGGAATTGCTT & 191 \\
\hline Phex & AGGCATCACATTCACCAACA & ATGGCACCATTGACCCTAAA & 103 \\
\hline Dmp1 & GGTITGACCTTGTGGGAAA & AATCACCCGTCCTCTCTTCA & 91 \\
\hline CapG & TCGGCATITCACAAGACAAC & GTTGGACTTCCACCACACC & 186 \\
\hline Sost & CGTGCCTCATCTGCCTACTT & ATAGGGATGGTGGGGAGGT & 185 \\
\hline
\end{tabular}

levels were normalized to those of glyceraldehyde-3-phosphate dehydrogenase (Gapdh), using the $2^{-\Delta \Delta C t}$ method.

\section{Immunostaining}

The spheroids were collected and fixed in the $4 \%$ paraformaldehyde. After washing samples with PBS, they were subjected to permeabilization using $0.1 \%$ triton X-100 for $30 \mathrm{~min}$, after which the samples were washed twice with PBS. Then, the samples were blocked using $4 \%$ bovine serum albumin at room temperature for $1 \mathrm{~h}$. After washing the blocking reagent away, we added the anti-DMP1 antibody (Abcam), and incubated the reaction mixture overnight at $4^{\circ} \mathrm{C}$. The samples were then washed twice with PBS and treated with the Alexa Flour 546 secondary antibody (Invitrogen), Alexa Fluor 488 Phalloidin (Invitrogen), and DAPI (Sigma) for $1 \mathrm{~h}$ at room temperature. After the samples were washed twice with PBS, they were preserved in PBS at $4^{\circ} \mathrm{C}$ until the time of observation. The stained samples were observed using the FLUOVIEW FV3000 (Olympus).

\section{Microarray}

We prepared RNA samples as described above, via the RTPCR method. The RNA samples were then examined using a spectrophotometer, to check their purity. After reverse transcription, followed by hybridization, the samples were analyzed by using the GeneChip Array (Mouse Genome 4302.0 Array), which is comprised of 34,000 genes.

In the individual experimental set, the raw data obtained using the spheroid samples were normalized to the monolayer sample. Relative gene expression levels were more than doubled or halved for some samples; these changes were considered to be modulated. Moreover, we only included samples whose $p$ value was lower than 0.05 . By sorting those modulated genes, we categorized and ranked them to assess the significance of modulated gene expression levels. To generate a heatmap of gene expression changes in the monolayer and spheroids, we utilized transcriptome analysis console (TAC) software (Thermo Fisher). From the resources section of DAVID Bioinformatics 6.8, we obtained the expression analysis systematic explorer (EASE) score for the enriched gene ontology according to the relevant biological process, cellular components, or molecular functions.

\section{Statistical Analysis}

The bars in the RT-PCR represent the means \pm standard error values. A student's $t$-test, one-way, or two-way ANOVA with Fisher's least significant difference (LSD) post-hoc test (with $\alpha=$ $0.05)$ were performed, to evaluate the statistical significance. If a $p$-value in the student's $t$-test is $<0.05$, we assumed that the difference was significant.

\section{RESULTS}

\section{The 3D Bone Spheroid Structure Highly Up-Regulated Osteocyte Markers Without Chemical Supplements}

Based on a previous study (Furukawa et al., 2001), we successfully established the method for fabricating bone spheroids using mouse pre-osteoblastic MC3T3-E1 cells, as illustrated in Figure 1A. After a 2-days incubation period, the monolayer and spheroids reconstructed using MC3TE-E1 cells were formed, as shown in Figures 1B,C. In Figures 1D,E and Table S1, their gene expression levels were then examined via RT-PCR. Osteoprogenitor, pre-osteoblast, and osteoblast markers between the monolayer and spheroids were compared in Figure 1D. In the spheroids, all these markers were significantly down-regulated, as compared to those in the monolayer condition, with the exception of Ocn. The osteoprogenitor markers Runx2 (0.64-fold change; $p<0.05)$ and $O s x(0.37$-fold change; $p<0.005)$ were significantly down-regulated. Moreover, the changes in the preosteoblast markers, such as Alp (0.35-fold change; $p<0.005)$ and Colla1 ( 0.11 -fold change; $p<0.005$ ) were also greatly decreased. Among the osteoblast markers, we examined the changes in the expression of $D l x 5, B s p$, and $O c n$. Whereas, the relative expression of Dlx5 (0.37-fold change; $p<0.005)$ and Bsp $(0.43$ fold change; $p<0.005)$ was significantly suppressed, the Ocn gene 


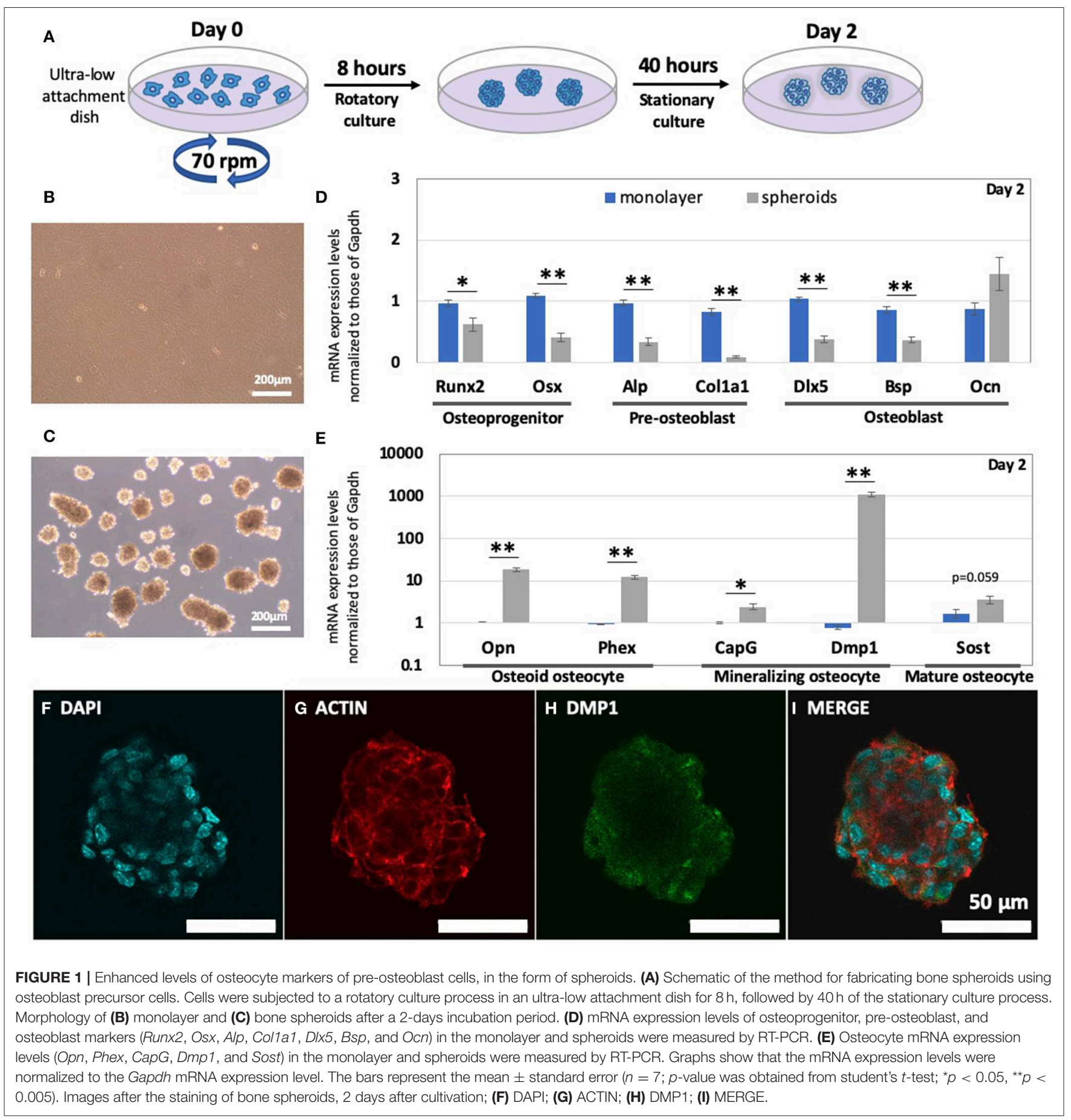

expression (1.65-fold change; $p=0.26$ ) was increased, though the level of increase was non-significant.

Conversely, osteocyte markers in the spheroids were greatly up-regulated, as compared to those in the monolayer, as represented in Figure 1E. The relative gene expression levels of osteoid osteocyte markers, including Opn (17.5-fold change; $p<0.005)$ and Phex (13.0-fold change; $p<0.005)$, were highly up-regulated. The spheroid structure also significantly up-regulated mineralizing osteocyte markers, such as CapG (2.37-fold change; $p<0.05)$ and Dmp1 (1,390-fold change; $p$ $<0.005)$. With regard to mature osteocyte markers, we also evaluated the genetic expression of Sost, and the relative change in its levels was up-regulated (2.13-fold change; $p=0.059)$. In addition, we conducted an immunofluorescence analysis of the spheroids in Figures 1F-I, to determine the levels of expression of the osteocyte protein DMP1. As a result, the DMP1 
protein expression levels inside the spheroid was confirmed within 2 days.

\section{Structural Effects on Osteocyte Markers Were Much Greater and Were Observed More Rapidly Than the Effects Attributable to Chemical Induction}

To evaluate the effects attributable to cell structure on preosteoblastic cells, as compared to those attributable to the use of chemicals, we included osteogenic supplements during cultivation and carried out RT-PCR. Figure 2A shows that the addition of osteogenic supplements in the monolayer for 2 days did not affect Runx2 (Two-way ANOVA: $p<0.005$ for monolayer vs. spheroids; $p=0.96$ for control vs. osteogenic supplements; $p=0.40$ for interaction), Osx (Two-way ANOVA: $p<0.005$ for monolayer vs. spheroids; $p=0.16$ for control vs. osteogenic supplements; $p=0.68$ for interaction), Alp (Two-way ANOVA: $p<0.005$ for monolayer vs. spheroids; $p=0.46$ for control vs. osteogenic supplements; $p=0.05$ for interaction), and Ocn (Two-way ANOVA: $p=0.61$ for monolayer vs. spheroids; $p=0.82$ for control vs. osteogenic supplements; $p=0.78$ for interaction) expression. However, the addition of chemical osteogenic supplements in the monolayer for 2 days slightly decreased Colla1 mRNA expression (Two-way ANOVA: $p<0.005$ for monolayer vs. spheroids; $p<0.05$ for control vs. osteogenic supplements; $p=0.06$ for interaction) and significantly up-regulated the expression of osteoblast markers, such as Dlx5 (Two-way ANOVA: $p<0.005$ for monolayer vs. spheroids; $p<0.05$ for control vs. osteogenic supplements; $p$ $=0.28$ for interaction) and Bsp (Two-way ANOVA: $p<0.005$ for monolayer vs. spheroids; $p<0.05$ for control vs. osteogenic supplements; $p<0.05$ for interaction). However, the addition of osteogenic supplements to the spheroids did not cause a significant change in the genetic expression of osteoprogenitor, pre-osteoblast, and osteoblast markers.

In Figure 2B, the osteocyte markers were then analyzed. The addition of osteogenic supplements did not significantly alter the genetic expression of osteocyte marker genes in the monolayer, such as Opn (Two-way ANOVA: $p<0.005$ for monolayer vs. spheroids; $p=0.64$ for control vs. osteogenic supplements; $p$ $=0.58$ for interaction), Phex (Two-way ANOVA: $p<0.005$ for monolayer vs. spheroids; $p=0.09$ for control vs. osteogenic supplements; $p=0.13$ for interaction), CapG (Two-way ANOVA: $p<0.005$ for monolayer vs. spheroids; $p=0.77$ for control vs. osteogenic supplements; $p=0.94$ for interaction), Dmp1 (Two way ANOVA: $p<0.005$ for monolayer vs. spheroids; $p$ $=0.11$ for control vs. osteogenic supplements; $p=0.11$ for interaction), and Sost (Two-way ANOVA: $p<0.05$ for monolayer vs. spheroids; $p=0.19$ for control vs. osteogenic supplements; $p$ $=0.12$ for interaction). However, on day 2 , the spheroid samples were subjected to chemical induction, which resulted in the upregulation of several osteocyte markers, including Phex (1.45fold change; $p<0.05)$ and Dmp1 (1.53-fold change; $p<0.05)$. Nonetheless, the results altogether showed that the changes in osteocyte gene expression induced by chemical induction were much less than those induced because of the structural effects.

\section{Cell Condensation in the 3D Structure Is Essential for Continued Osteocyte Differentiation}

To evaluate the significance of the cell condensation induced by the 3D spheroid structure on the MC3T3-E1 cells, we introduced a new model, i.e., cells derived from the spheroids. To prepare this model, spheroids were cultured in the ultra-low attachment dish for 2 days, and then transferred to a standard culture dish, as described in Figure 3A. In comparison with this model, we utilized the 2D monolayer and 3D spheroids incubated for 4 days, which are shown in Figures 3B,C, respectively. The cells derived from spheroids became attached onto the standard culture dish and became spread out over the culture dish immediately after the spheroids were transferred to the culture dish. After a 2-days period of stationary culture, the spheroids almost lost their 3D structure and formed a 2D monolayer, as described in Figure 3D.

As shown by RT-PCR in Figures 3E,F, the gene expression levels of osteoprogenitor [Osx (One way ANOVA: $p<0.005$ )], pre-osteoblast [Alp (One way ANOVA: $p<0.005$ ) \& Colla1 (One way ANOVA: $p<0.005)]$, and osteoblast markers $[D l x 5$ (One way ANOVA: $p=0.16$ ) \& Bsp (One way ANOVA: $p<$ $0.005)]$ in cells derived from the spheroids were similar level to those for spheroids incubated for 4 days. However, osteocyte markers, including Opn (One way ANOVA: $p<0.005$ ), Phex (One way ANOVA: $p<0.05$ ), CapG (One way ANOVA: $p<$ 0.005 ), Dmp1 (One way ANOVA: $p<0.05$ ), and Sost (One way ANOVA: $p<0.05$ ) showed were down-regulated up to the level of the monolayer in cells derived from the spheroids.

\section{Cell Condensation Achieved From the 3D Spheroid Structure Modulated the Clustering of Enriched Gene Expression During the ECM-Receptor Interaction, Cell Proliferation, Cell Cycle, Hypoxia, and Ossification}

In order to investigate all the changes in gene expression in the spheroids, as compared to those in the monolayer, we performed a microarray analysis. Figure 4 shows the heatmap of mRNAs, in accordance with the gene ontology, (A) ECM-receptor interaction, (B) cell proliferation, (C) cell cycle, (D) hypoxia, and (E) ossification. ECM-receptor interaction was significantly activated in the spheroids, as shown in Figure 4A. The results showed that the enrichment of ECM occurred because the 3D structure caused significant changes in integrins (Itga6, Itgb3, Itga10, Itga3, Itgb2, Itgb8, Itga5) as well as the up-regulation of Fn1 or Dab2.

The expression of many cell proliferation-related genes, including E2f1, Bub1, Aurka, and Mki67 was significantly suppressed in spheroids, as shown in Figure 4B. With regard to the gene ontology (GO) term, over-represented categories of functional genes were ranked by their EASE scores (geneenrichment), as shown in Table S2. The highest and second highest EASE scores represented cell cycle and cell division, respectively. A significant down-regulation of DNA replicationrelated genes (Dna2, Ccna2, Gmnn, etc.), M phase-related genes 


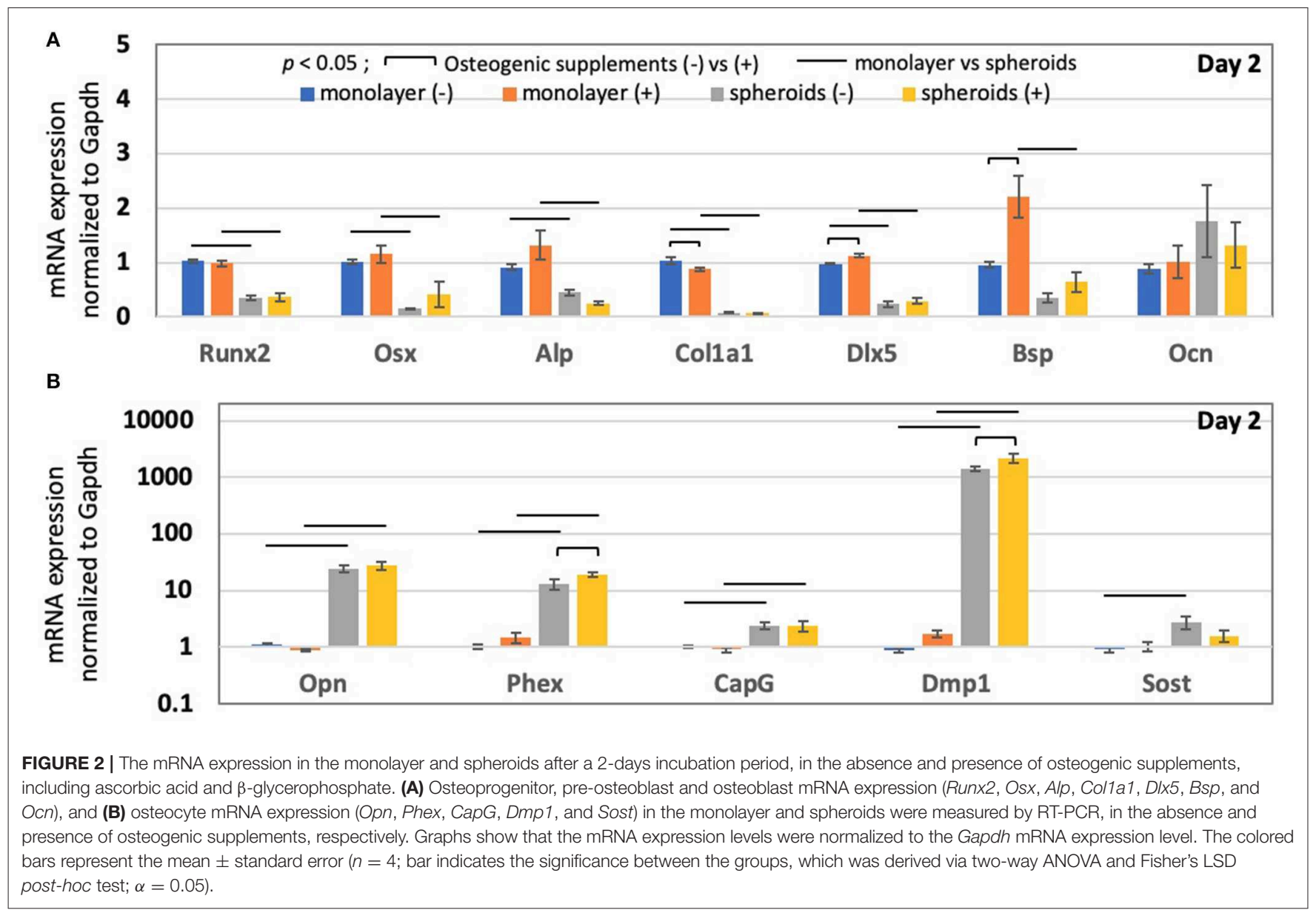

(Ccnb1), and G1 phase-related genes (Mcm10, Mcm8, Mcm5, Mcm3, Mcm2, Mcm4, Mcm6, etc.) was observed, as shown in Figure 4C, whereas cell expression levels of cycle arrest (G1/G2 phase arrest) genes, including Ccng2, were significantly altered. In addition, hypoxia-related genes, including Vegf in Figure 4D were significantly modulated in the spheroids, as compared to the level of modulation observed in the monolayer. The ossification markers including the osteocyte markers, matrix metalloproteinase $(\mathrm{Mmp})$ family (Prideaux et al., 2015), and Tgfb1 (Janssens et al., 2005) are shown in Figure 4E. They resulted in the up-regulation of osteocyterelated markers (Mmp2, Mmp11, Mmp14, Mmp13, Mmp19, Tgfb1, CapG, Phex, Dmp1, etc.) as well as down-regulation of osteoblast-related markers (Col8a1, Col1a1, Dlx5, Igf1, etc.), which was consistent with the RT-PCR results. The genes that were the most significantly up- and down-regulated in the spheroids, as compared to the monolayer were $\operatorname{Dmpl} 1$ and Colsa1, respectively.

\section{DISCUSSION}

In this study, we successfully developed the $3 \mathrm{D}$ cell structures using mouse pre-osteoblast cells, known as bone spheroids. Because we hypothesized that the cell condensation plays a significant role in osteogenesis differentiation, we utilized $3 \mathrm{D}$ spheroid models, which enabled us to evoke the cell condensation. As a result, we found out that osteogenic differentiation into osteocyte-like cells in the spheroid was remarkably promoted, as compared to that in the $2 \mathrm{D}$ monolayer model, without using chemical supplements. In Figure 1, most of the pre-osteoblast or osteoblast markers in spheroids were significantly suppressed in 2 days, whereas the osteocyte markers (Opn, Phex, CapG, Dmp1, and Sost) were highly up-regulated. The increase in Dmp1 gene expression was especially significant, as it was an $\sim 1,400$-fold change, as shown by both RT-PCR and microarray analysis, because Dmp1 is regarded as an osteocyte specific marker that modulates osteocyte formation (Kalajzic et al., 2004; Lu et al., 2011). This level of change in Dmp1 gene expression, achieved in the form of spheroids, has not been reported with the conventional $2 \mathrm{D}$ model, even after long periods of cell culture with chemical supplements. Moreover, the results of immunostaining analysis confirmed the level of expression of the DMP1 protein in the spheroid. Since the matured osteocyte marker, Sost, was modestly up-regulated, pre-osteoblast cells in the spheroids appeared similar to cells at a more immature stage of osteocyte differentiation. Nevertheless, it is still satisfactory to note that our model enables us to trigger and accelerate the in vitro process of osteocyte differentiation within a short 

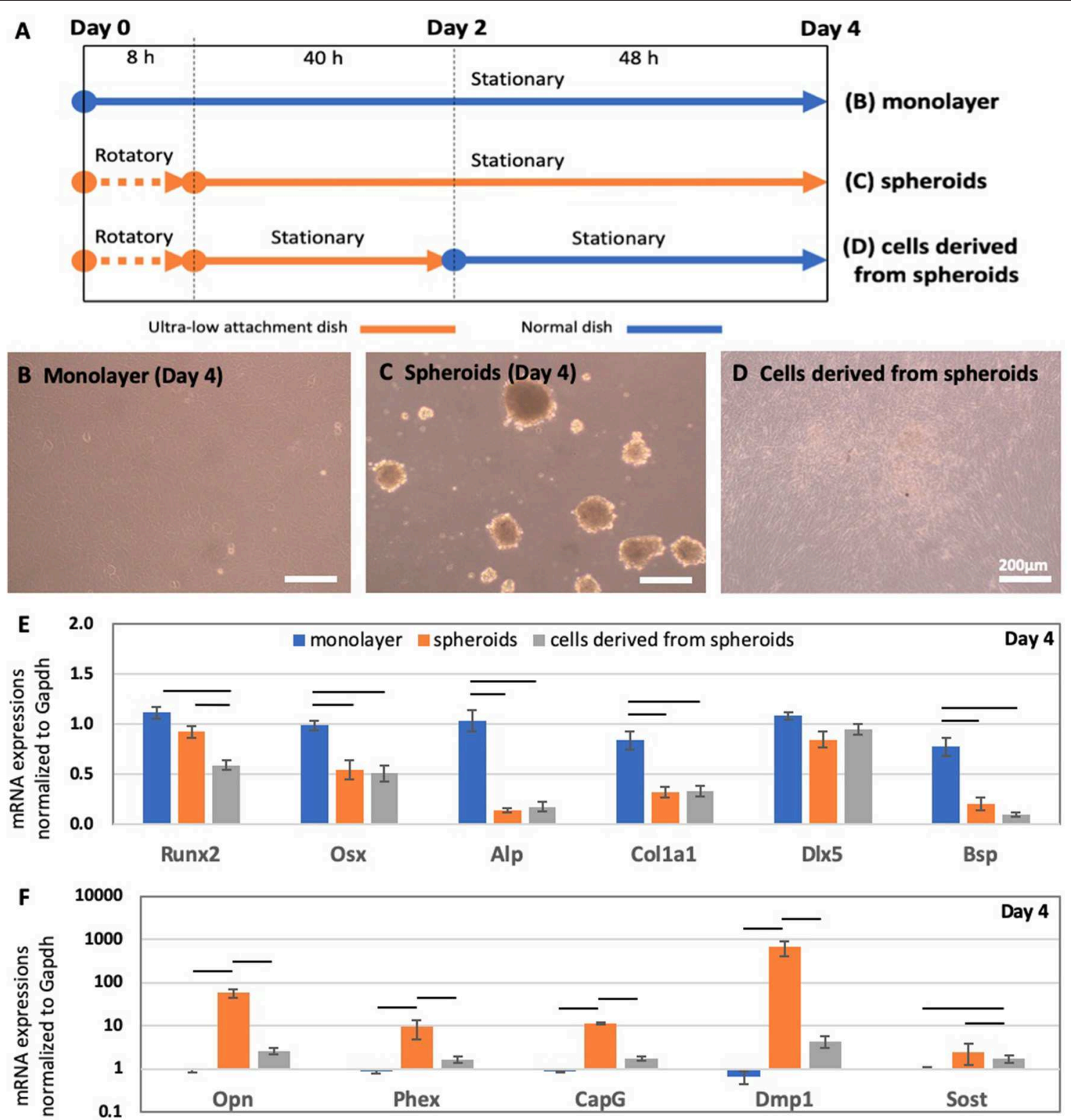

FIGURE 3 | (A) Schematic of the experimental timeline for deriving the monolayer, spheroids, and spheroid-derived cells after a 4-days incubation period. Morphology of osteoblast precursor cells in the form of the (B) monolayer and (C) spheroids after a 4-days incubation period. (D) Cells derived from spheroids, after spheroids were transferred onto a culture dish on day 2. After incubating them for 2 days in the ultra-low attachment dish, the spheroids were transferred into a culture dish and incubated further for 2 days. RT-PCR analysis of the mRNA expression of (E) pre-osteoblast and osteoblast markers (Runx2, Osx, Alp, Col1a1, D/x5, and Bsp) and (F) osteocyte markers (Opn, Phex, CapG, Dmp1, and Sost) in the monolayer, spheroids, and spheroid-derived cells. Graphs show that the mRNA expression levels were normalized to Gapdh mRNA expression levels. The colored bars represent the mean \pm standard error $(n=6$; bar indicates the significance between the groups, which was derived via one-way ANOVA with Fisher's LSD post-hoc test; $\alpha=0.05$ ).

period of 2 days, which is greater than that for the chemical induction process.

With regard to the process of bone nodule formation observed in the 2D in vitro model (Alami et al., 2016; Kawai et al., 2019), it is known that its 3D dome-shaped structure was formed after the subcultured cells became confluent and led to the cell condensation. As shown in a previous study (Maniatopoulos et al., 1988), the osteocyte markers derived from pre-osteoblasts to which osteogenic supplements were added were expressed only inside the bone nodule over 3-4 weeks. The up-regulation of osteomarkers in the spheroids seemed to be a similar phenomenon induced by highly condensed cell culture conditions. This suggested that each of the spheroids in our model might mimic the bone nodule. Interestingly, our model did not even require any chemical induction for the significant changes in the osteoblast 


\section{A ECM-receptor interaction}

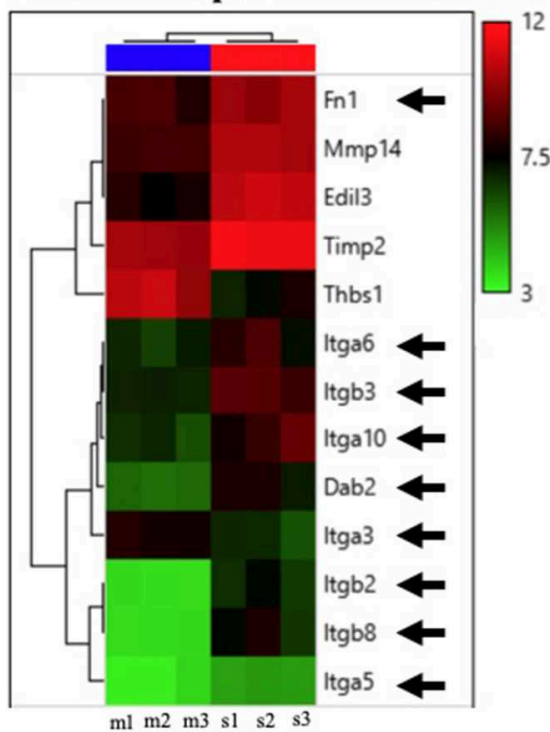

D Hypoxia

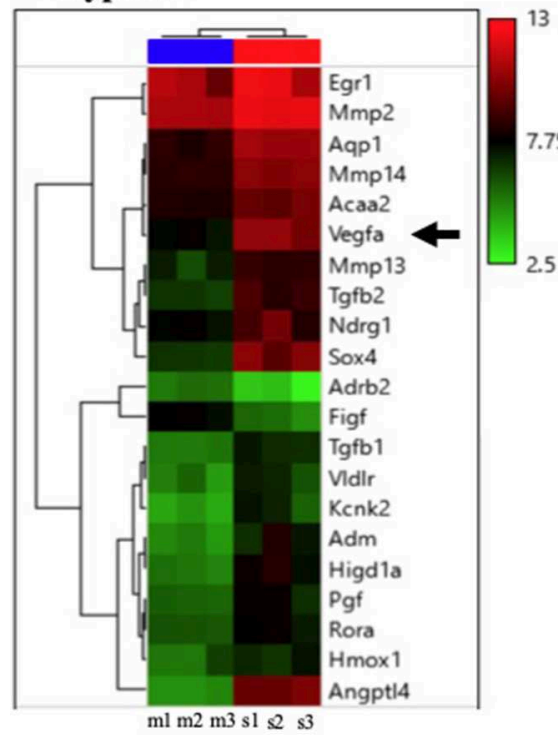

\section{B Cell proliferation}

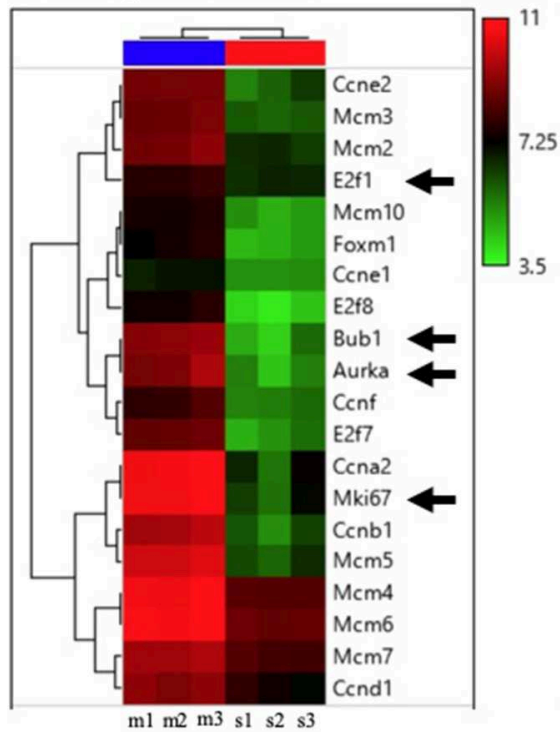

E Ossification

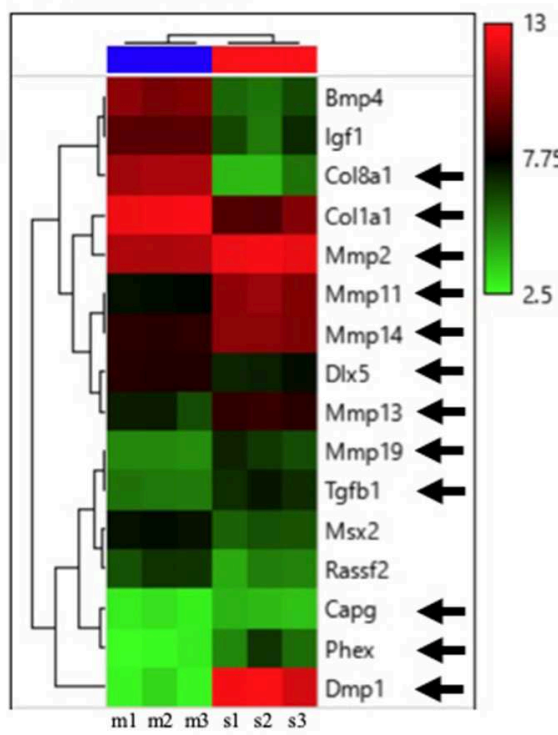

\section{c Cell cycle}

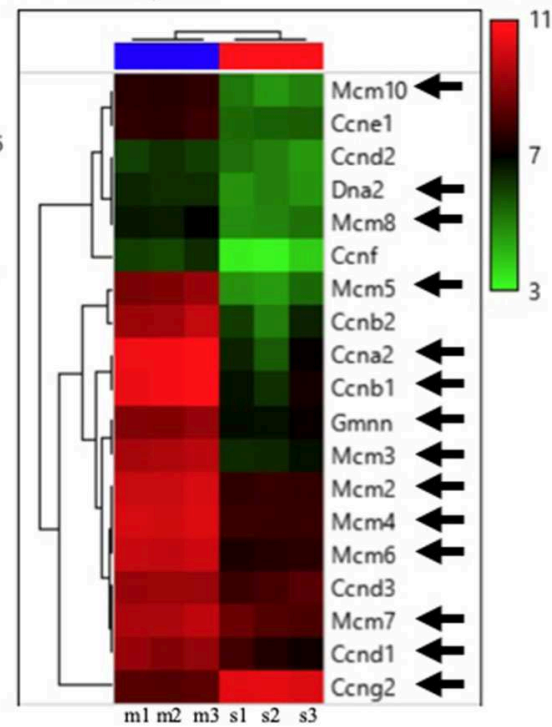

FIGURE 4 | Heatmaps of significantly modulated genes enriched in (A) ECM-receptor interaction, (B) cell proliferation, (C) cell cycle, (D) hypoxia, and (E) ossification. The relative gene expression levels, for which the fold-changes were above 2.0 or below 0.5 , and whose $p$-value was lower than 0.05 , were selected ( $n=3$ ).

and osteocyte markers to occur in the short-term period, within 2 days.

We then carried out the experiments in the presence of the osteogenic supplements, to evaluate the effect of chemical induction on the spheroids and compare the chemical effects to the structural 3D effects. Though osteogenic supplements were added, as shown in Figure 2, the culture period for the monolayer was not enough to induce significant changes in the osteoblast or osteocyte markers within 2 days, although the levels of several osteoblast markers, including Dlx5 and Bsp in the monolayer were slightly increased. However, the osteogenic supplements helped to further promote Phex and Dmpl gene expression in the spheroids, but the effects attributable to chemicals were not as much as the structural effects caused because of 3D structure formation. These results imply that the osteocyte differentiation of pre-osteoblast cells strongly depends on the 3D structural effect, because the cell condensation was enhanced beyond those observed with chemical induction.

To determine the significance of cell condensation for osteocyte differentiation, we transferred spheroids from the ultralow attachment dish to a standard culture dish after a 2-days incubation period, and allowed the spheroids to be cultured for an additional 2 days. As a result, the spheroids lost their 3D structure and were converted into a $2 \mathrm{D}$ monolayer, as shown in Figure 3. This implies that the spheroids formed for 2 days in the ultra-low attachment dish were still active enough for 
the cell-substrate interaction to occur, for their attachment to the standard plastic cell culture dish. Interestingly, cells derived from the spheroids were spread out over the culture dish, and the expression of their osteocyte genes as spheroids was reversed to the level of a monolayer cultured for 4 days. Hence, the up-regulation of osteocyte genes of pre-osteoblast cells can be maintained, when the cells exhibit the cell condensation observed in the 3D structure. Thus, osteocyte differentiation from pre-osteoblast or osteoblast cells was triggered by the cell condensation, whereas cells at lower cell condensation levels become more inactive with regard to the induction of osteocyte differentiation.

Due to the structural environment of spheroids, the cell condensation for pre-osteoblast cells was induced inside the spheroids, and this eventually brought about an increase in the ECM secreted by surrounding cells. Because of microarray analysis in Figure 4, ECM accumulation induced intracellular signaling pathways in the surrounded cells via integrins. The results of microarray analysis also showed that the cell condensation achieved in the spheroid caused a cessation in cell proliferation and simultaneously allowed cells to induce cell cycle arrest. These events altogether might cause the pre-osteoblast cells in the spheroids to become terminally differentiated into osteocyte-like cells. Moreover, hypoxia was thought to contribute to the promotion of osteocyte differentiation inside spheroids, as hypoxic conditions are known to induce osteocytogenesis differentiation (Hirao et al., 2007; Zahm et al., 2008). The up-regulation of hypoxia-related markers, including Vegf was confirmed in the spheroids. This occurred because the cells inside the spheroid were subjected to hypoxic conditions instead of monolayer conditions, which enhanced the upregulation of osteocyte markers further. We think that several potential factors could induce the osteocyte differentiation after cell condensation; however, further studies, including those regarding the cell-cell interaction force, cytoskeletal tension, or cell shape would be required to understand the mechanism in detail.

As illustrated in Figure 5, we showed that it is more efficient and essential for pre-osteoblast cells in the highly condensed 3D structure to trigger and facilitate differentiation into osteocytelike cells in vitro. To date, the osteoblast is only thought to line up two-dimensionally on the bone at a targeted site, where the bone remodeling occurs in vivo. Our results altogether suggest that osteoblasts achieve the ability to differentiate into osteocytelike cells, after they proliferate and become condensed at the targeted site of bone remodeling. For several decades, researchers have attempted to induce osteocyte differentiation over 1-2 months, by culturing cells to ensure that they are over-confluent and condensed, until the formation of the $3 \mathrm{D}$ bone nodule, in combination with chemical supplements (Bhargava et al., 1988; Maniatopoulos et al., 1988; Schecroun and Delloye, 2003). However, this study showed that osteocyte differentiation highly depends upon cell condensation levels that are beyond those attributable to a longer period of chemical induction. Although it is extremely difficult to observe this event in vivo, its occurrence would help us to narrow the gap between in vitro and in vivo bone remodeling. Hence, we first highlighted the significance of a highly condensed $3 \mathrm{D}$ structure, in which pre-osteoblast or osteoblast cells would be induced to differentiate into osteocytelike cells. Our group has also developed a 3D tissue engineered construct of a different type, which was reconstructed using only pre-osteoblast cells (MC3T3-E1), without the introduction of any biomaterial. It rendered the changes in gene expression levels similar to those observed in the present study, with regard to the up-regulation in the osteocyte marker levels and downregulation in the osteoblast marker levels. These results together reveal the significance of cell condensation, and the manner in which the pre-osteoblast cells are triggered to undergo osteocyte

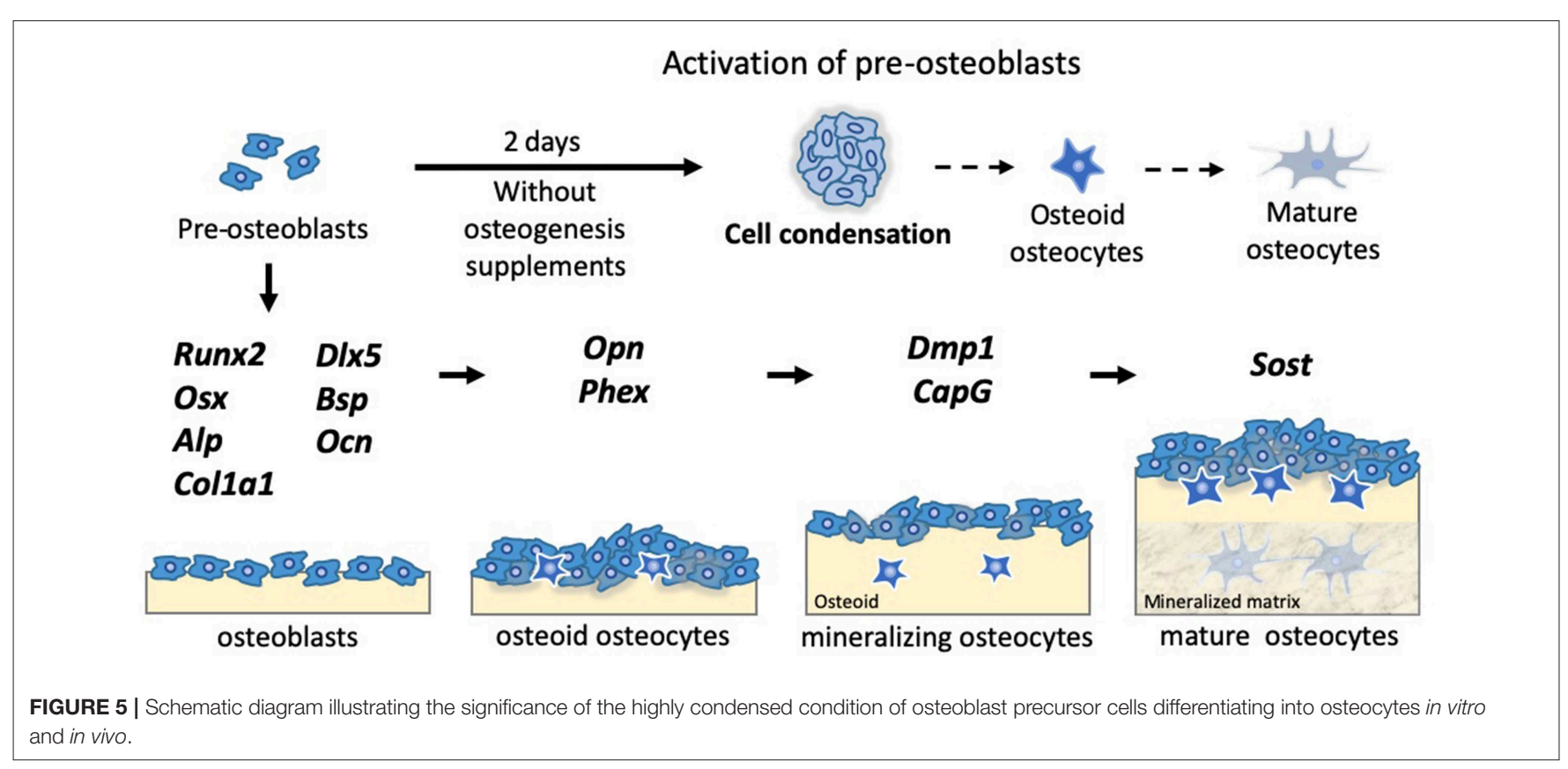


differentiation. In this study, we utilized murine osteoblast precursor cells, which are well-studied and widely used cell lines in bone research; however, further research needs to be conducted to support our findings using a different cell line or primary cells, such as stem cells. Moreover, it will be meaningful to carry out experiments involving bone spheroids over a longer period, to determine further changes in their functioning or histology as osteocytes.

Here, we first established a novel method to fabricate 3D bone spheroids using mouse precursor osteoblast cells under rotatory culture conditions, by changing several parameters, including the cell density or rotational speed, and the time set for the rotatory shaker, based on the parameters described in the previous study (Furukawa et al., 2001). Although several studies attempted to fabricate the $3 \mathrm{D}$ in vitro structure for generating the bone model that enhanced osteogenic differentiation, they utilized scaffoldbased techniques, and used biomaterials, such as collagen or agar-agar gel (Restle et al., 2015; Zujur et al., 2017). Moreover, these models could slightly enhance osteocyte differentiation with the accompanying process of osteoblast differentiation, because of which the factor triggering independent osteocyte differentiation remained unknown. For biomedical applications using a tissue engineering approach, these mediating materials might carry the risk of affecting cell behavior or inducing negative interactions with donor cells after transplantation. Because our bone spheroids did not recruit any artificial materials during the fabrication process, they are predicted to become a more appropriate model for clinical use. Particularly, our method allowed the mass production of spheroids, whereas spheroids are conventionally generated one by one in a tube or well. This method can be optimized and applied to fabricate spheroids for various cell types, by adjusting the cell density or rotatory culture period, because the strength of cell-cell interactions or cell-ECM interactions differ for varied types of cells. Furthermore, the 3D in vitro structures for other cell models could enhance their differentiation capability, as shown in this study.

In the field of bone tissue engineering, mesenchymal stem cells (MSC), embryonic stem cells (ESC), or induced pluripotent stem cell (iPSC) reportedly participate in the repair of osseous defects, such as traumatic fractures, osteoarthritis, or rheumatoid arthritis (Amini et al., 2012; Goonoo and Bhaw-Luximon, 2018). Moreover, MSC treatment for large bone defects or the severe osteogenesis imperfecta model was provided in several studies (Horwitz et al., 1999; Quarto et al., 2009). However, the limited differentiation capacity of MSCs remains a concern observed for current clinical therapies, whereas the utilization of more differentiated cells is thought to be safer. The use of our model allows the mass production of differentiated spheroids in vitro, in combination with osteogenic supplements, and further enables us to transplant them to a targeted site via a syringe injection.

\section{REFERENCES}

Adachi, T., Aonuma, Y., Tanaka, M., Hojo, M., Takano-Yamamoto, T., and Kamioka, H. (2009). Calcium response in single osteocytes to locally applied mechanical stimulus: differences in cell process and
Several applications of spheroids can be expected to be developed in the bone tissue engineering field.

In conclusion, this study provides new insights into the significance of the cell condensation conditions achieved via the $3 \mathrm{D}$ structure, to enable the osteoblast precursor cells to facilitate differentiation into osteocyte-like cells. Our 3D bone spheroid model, which was generated using mouse osteoblast precursor cells, demonstrated the significant up-regulations in the osteocyte markers within 2 days. Consequently, we suggested that cell condensation is the key factor triggering greater levels of in vitro osteocyte differentiation than that observed with chemical induction.

\section{DATA AVAILABILITY STATEMENT}

All datasets generated for this study are included in the manuscript/Supplementary Files.

\section{AUTHOR CONTRIBUTIONS}

JK and TA contributed to the study design, data collection, manuscript writing, and final approval of the manuscript.

\section{FUNDING}

This work was supported by Advanced Research and Development Programs for Medical Innovation (AMEDCREST), by elucidation of mechanobiological mechanisms and their application to the development of innovative medical instruments and technologies from Japan Agency for Medical Research and Development (AMED), by the acceleration program for intractable diseases research utilizing diseasespecific iPS cells from AMED, and by the Grant-in-Aid for Research Activity Start-up (19K23604) from Japan Society for the Promotion of Science (JSPS).

\section{ACKNOWLEDGMENTS}

We would like to thank Junko Sunaga for providing technical support to us.

\section{SUPPLEMENTARY MATERIAL}

The Supplementary Material for this article can be found online at: https://www.frontiersin.org/articles/10.3389/fbioe. 2019.00288/full\#supplementary-material

Table S1 | Expression levels of genes in monolayer and spheroids after 2 days cultivation. The average Ct values were exhibited in the table.

Table S2 | Over-represented gene functional categories with EASE scores (gene-enrichment).

cell body. J. Biomech. 42, 1989-95. doi: 10.1016/j.jbiomech.2009. 04.034

Alami, S. M., Gangloff, S. C., Laurent-Maquin, D., Wang, Y., and Kerdjoudj, H. (2016). Concise review: in vitro formation of bonelike nodules sheds light on the application of stem cells for bone 
regeneration. Stem Cells Transl. Med. 5, 1587-1593. doi: 10.5966/sctm. 2015-0413

Amini, A. R., Laurencin, C. T., and Nukavarapu, S. P. (2012). Bone tissue engineering: recent advances and challenges. Crit. Rev. Biomed. Eng. 40, 363-408. doi: 10.1615/CritRevBiomedEng.v40.i5.10

Bhargava, U., Bar-Lev, M., Bellows, C. G., and Aubin, J. E. (1988). Ultrastructural analysis of bone nodules formed in vitro by isolated fetal rat calvaria cells. Bone 9, 155-63. doi: 10.1016/8756-3282(88)90005-1

Blair, H. C., Larrouture, Q. C., Li, Y., Lin, H., Beer-Stoltz, D., Liu, L., et al. (2017). Osteoblast differentiation and bone matrix formation in vivo and in vitro. Tissue Eng. Part B Rev. 23, 268-280. doi: 10.1089/ten.teb.2016.0454

Bonewald, L. F. (2011). The amazing osteocyte. J. Bone Miner. Res. 26, 229-38. doi: $10.1002 /$ jbmr.320

Buttery, L. D. K., Bourne, S., Xynos, J. D., Wood, H., Hughes, F. J., Hughes, S. P. F., et al. (2004). Differentiation of osteoblasts and in vitro bone formation from murine embryonic stem cells. Tissue Eng. 7, 89-99. doi: 10.1089/107632700300003323

Capulli, M., Paone, R., and Rucci, N. (2014). Osteoblast and osteocyte: Games without frontiers. Arch. Biochem. Biophys. 561, 3-12. doi: 10.1016/j.abb.2014.05.003

Coelho, M. J., and Fernandes, M. H. (2000). Human bone cell cultures in biocompatibility testing. Part II: effect of ascorbic acid, b-glycerophosphate and dexamethasone on osteoblastic differentiation. Biomaterials 21, 1095-1102. doi: 10.1016/S0142-9612(99)00192-1

Dye, B. R., Hill, D. R., Ferguson, M. A. H., Tsai, Y., Nagy, M. S., Dyal, R., et al. (2015). In vitro generation of human pluripotent stem cell derived lung organoids. Elife 4:1999. doi: 10.7554/eLife.05098

Furukawa, K. S., Ushida, T., Sakai, Y., Suzuki, M., Tanaka, J., and Tateishi, T. (2001). Formation of human fibroblast aggregates (spheroids) by rotational culture. Cell Transplant. 10, 441-45. doi: 10.3727/000000001783986503

Goonoo, N., and Bhaw-Luximon, A. (2018). Regenerative medicine: induced pluripotent stem cells and their benefits on accelerated bone tissue reconstruction using scaffolds. J. Mater. Res. 33, 1573-91. doi: 10.1557/jmr.2018.132

Hirao, M., Hashimoto, J., Yamasaki, N., Ando, W., Tsuboi, H., Myoui, A., et al. (2007). Oxygen tension is an important mediator of the transformation of osteoblasts to osteocytes. J. Bone Miner. Metabo. 25, 266-76. doi: 10.1007/s00774-007-0765-9

Horwitz, E. M., Prockop, D. J., Fitzpatrick, L. A., Koo, W. W. K., Gordon, P. L., Neel, M., et al. (1999). Transplantability and therapeutic effects of bone marrow-derived mesenchymal cells in children with osteogenesis imperfecta. Nat. Med. 5, 309-13. doi: 10.1038/6529

Janssens, K., Dijke, P., Janssens, S., and Hul, W. V. (2005). Transforming growth factor-betal to the bone. Endocr. Rev. 26, 743-774. doi: 10.1210/er.2004-0001

Kalajzic, I., Braut, A., Guo, D., Jiang, X., Kronenberg, M. S., Mina, M., et al. (2004). Dentin matrix protein 1 expression during osteoblastic differentiation, generation of an osteocyte GFP-transgene. Bone 35, 74-82. doi: 10.1016/j.bone.2004.03.006

Kawai, S., Yoshitomi, H., Sunaga, J., Alev, C., Nagata, S., Nishio, M., et al. (2019). In vitro bone-like nodules generated from patient-derived iPSCs recapitulate pathological bone phenotypes. Nat. Biomed. Eng. 3, 558-70. doi: 10.1038/s41551-019-0410-7

Lu, Y., Yuan, B., Qin, C., Cao, Z., Xie, Y., Dallas, S. L., et al. (2011). The biological function of DMP-1 in osteocyte maturation is mediated by its $57-\mathrm{kDa}$ c-terminal fragment. J. Bone Miner. Res. 26, 331-40. doi: 10.1002/jbmr.226

Malaval, L., Modrowski, D., Gupta, A. K., and Aubin, J. E. (1994). Cellular expression of bone-related proteins during in vitro osteogenesis in rat bone marrow stromal cell cultures. J. Cell. Physiol. 158, 555-72. doi: $10.1002 /$ jcp. 1041580322

Maniatopoulos, C., Sodek, J., and Melcher, A. H. (1988). Bone formation in vitro by stromal cells obtained from bone marrow of young adult rats. Cell Tissue Res. 254, 317-30. doi: 10.1007/BF00225804
McCracken, K. W., Catá, E. M., Crawford, C. M., Sinagoga, K. L., Schumacher, M., Rockich, B. E., et al. (2014). Modelling human development and disease in pluripotent stem-cell-derived gastric organoids. Nature 516, 400-404. doi: 10.1038/nature13863

Nair, A. K., Gautieri, A., Chang, S., and Buehler, M. J. (2013). Molecular mechanics of mineralized collagen fibrils in bone. Nat. Commun. 4:1724. doi: $10.1038 /$ ncomms 2720

Prideaux, M., Staines, K. A., Jones, E. R., Pitsillides, A. A., and Farquharson, C. (2015). MMP and TIMP temporal gene expression during osteocytogenesis. Gene Expr. Patterns 18, 29-36. doi: 10.1016/j.gep.2015. 04.004

Quarles, L. D., Yohay, D. A., Lever, L. W., Caton, R., and Wenstrup, R. J. (1992). Distinct proliferative and differentiated stages of murine MC3T3-E1 cells in culture: an in vitro model of osteoblast development. J. Bone Miner. Res. 7, 683-92. doi: 10.1002/jbmr.5650070613

Quarto, R., Mastrogiacomo, M., Cancedda, R., Kutepov, S. M., Mukhachev, V., Lavroukov, A., et al. (2009). Repair of large bone defects with the use of autologous bone marrow stromal cells. N Engl J Med. 344, 385-86. doi: 10.1056/NEJM200102013440516

Restle, L., Costa-Silva, D., Lourenço, E. S., Bachinski, R. F., Batista, A. C., Linhares, A. B. R., et al. (2015). A 3D osteoblast in vitro model for the evaluation of biomedical materials. Adv. Mater. Sci. Eng. 2015, 1-8. doi: 10.1155/2015/268930

Rossi, G., Manfrin, A., and Lutolf, M. P. (2018). Progress and potential in organoid research. Nat. Rev. Genet. 19, 671-687. doi: 10.1038/s41576-018-0051-9

Sasai, Y. (2013). Cytosystems dynamics in self-organization of tissue architecture. Nature 493, 318-26. doi: 10.1038/nature11859

Schecroun, N., and Delloye, C. (2003). Bone-like nodules formed by human bone marrow stromal cells: comparative study and characterization. Bone 32, 252-60. doi: 10.1016/S8756-3282(02)00970-5

Spence, J. R., Mayhew, C. N., Rankin, S. A., Kuhar, M. F., Vallance, J. E., Tolle, K., et al. (2011). Directed differentiation of human pluripotent stem cells into intestinal tissue in vitro. Nature 470, 105-9. doi: 10.1038/nature 09691

Takebe, T., Sekine, K., Enomura, M., Koike, H., Kimura, M., Ogaeri, T., et al. (2013). Vascularized and functional human liver from an iPSC-derived organ bud transplant. Nature 499, 481-84. doi: 10.1038/nature12271

Wang, D., Christensen, K., Chawla, K., Xiao, G., Krebsbach, P. H., and Franceschi, R. T. (1999). Isolation and characterization of MC3T3-E1 preosteoblast subclones with distinct in vitro and in vivo differentiation/mineralization potential. J. Bone Miner. Res. 14, 893-903. doi: 10.1359/jbmr.1999.14. 6.893

Weatherholt, A. M., Fuchs, R. K., and Warden, S. J. (2012). Specialized connective tissue: bone, the structural framework of the upper extremity. J. Hand Ther. 25, 123-32. doi: 10.1016/j.jht.2011.08.003

Zahm, A. M., Bucaro, M. A., Srinivas, V., Shapiro, I. M., and Adams, C. S. (2008). Oxygen tension regulates preosteocyte maturation and mineralization. Bone 43, 25-31. doi: 10.1016/j.bone.2008.03.010

Zujur, D., Kanke, K., Lichtler, A. C., Hojo, H., Chung, U., and Ohba, S. (2017). Three-dimensional system enabling the maintenance and directed differentiation of pluripotent stem cells under defined conditions. Sci. Adv. 3:e1602875. doi: 10.1126/sciadv.1602875

Conflict of Interest: The authors declare that the research was conducted in the absence of any commercial or financial relationships that could be construed as a potential conflict of interest.

Copyright (c) $2019 \mathrm{Kim}$ and Adachi. This is an open-access article distributed under the terms of the Creative Commons Attribution License (CC BY). The use, distribution or reproduction in other forums is permitted, provided the original author(s) and the copyright owner(s) are credited and that the original publication in this journal is cited, in accordance with accepted academic practice. No use, distribution or reproduction is permitted which does not comply with these terms. 